\section{Physician participation needed in AIDS education}

The acquired immune deficiency syndrome (AIDS) is unlike any other disease or syndrome that we have previously experienced. Humanity has embarked on a mortal race with a novel pathogen of great subtlety. By its very nature, AIDS raises both unnecessary fear and unwarranted complacency. Its inherent complexity and the rapid addition of new information often leads to misunderstandings. Generalizations are often taken out of context and used inappropriately.

However, in the area of prevention, the message from the United States Public Health Service has been consistent. Information contained in the "Surgeon General's Report on AIDS" issued almost a year ago remains valid today. Our single defense against this disease from the very beginning has been appropriate information and education.

As physicians, we must assume the leadership role in education about AIDS, not only for our patients, but also for ourselves and the general public. Beginning with this issue, the JAOA initiates a series of articles directed toward AIDS prevention and control. This is an important educational effort, as I am aware that approximately 25,000 osteopathic physicians are involved daily in the primary care of tens of thousands of patients. As the Surgeon General of the United States Public Health Service, I ask that you join with me in assuming this leadership role for the benefit of mankind.

A focus on prevention of the sexual transmission of AIDS must be pursued because there is neither a vaccine nor a cure for this disease. It is difficult for all of us to publicly discuss sexual issues, but we have no choice when the alternative is almost certain death.

In the prevention of AIDS, an important message has been lost or at least minimized because of the media's emphasis on the use of condoms. This message is rather simple. For those who abstain or who have a mutually faithful monogamous sexual relationship, AIDS presents no problem. However, for sexually active individuals with multiple partners, there is no choice but to encourage the use of condoms. Therefore, it becomes imperative that physicians make themselves knowledgeable about the appropriate use and limitations of condoms.

The Food and Drug Administration is moving quickly to ensure that the condom industry complies with the standard that no more than four condoms per thousand should fail the leakage test. However, even this low leakage incidence is theoretically unacceptable. Consequently, based on reports presented at the Third International Conference on AIDS, I have also recommended that condoms be supplemented by lubrication with a jelly or cream containing at least $65 \mathrm{mg}$. of nonoxynol-9, which has been suggested as helpful in killing the virus.

There are other considerations that must be discussed with your patients concerning condoms. Only latex condoms should be used because natural membrane products may not prevent viral transmission. The proper use of condoms must also be stressed because many people do not know how to use this preventative device appropriately. Condoms should be rolled onto the erect penis prior to contact with the sexual partner's body. The penis should be withdrawn from the partner before erection is lost. Finally, a new condom should be used prior to each sexual act.

The patient should be dealt with kindly in a concerned and nonjudgmental fashion. Questions from your patients may signal deep seated fears that are not justifiable. Patients must be told that AIDS cannot be transmitted by casual nonsexual contact. AIDS is not an easily transmittable disease. Advise patients to abstain from sex with people they do not know with certainty to be uninfected with the virus. Abstinence from sex with persons other than seronegative partners is of paramount importance. The seronegative partner should not have had intercourse six months before, or anytime after, the date of the negative test. Their partners also must have abstained from having sex with other people as well.

If the infectiousness is in doubt, a blood test should be performed after discussing the meaning of the test with the patient. Patients should be in- 
formed that confidentiality on the part of the physician will be ensured. Sharing the information will occur only with other professionals who need to know. When appropriate, the physician should discuss the advisability of notifying a spouse or other sexual contacts. A woman who tests positively for the antibodies to the human immunodeficiency virus (HIV) should be advised that AIDS may be passed to any child she conceives.

It is necessary to stress the importance of being cautious of people who belong to organizations that offer "safe sex" simply because they issue a health card. Generally these health cards are out of date, and one has to question why such a person has a card like this in the first place. Tell your patients that if they have doubts about their sexual partner, they should use a condom. Additionally, patients should be informed not to practice anal intercourse, because the rectal mucosa bleeds readily, providing easy access for the virus to enter the body.

Given the background and considerations, I believe most physicians would agree AIDS is the most pressing public health problem in America today. This disease will affect each and every one of us. For those physicians who have not had an experience with this problem in their own practice, it is safe to suggest that they will, at least, be affected indirectly by the sheer economic impact of this crisis. If the public will heed and practice the above advice, I believe that the AIDS epidemic can be contained. If this advice is ignored, this disease will be with us for a very long time.

We must stop the spread of AIDS. The people of America need your full attention and dedication. The lives of people at risk, including unsuspecting young people and the unborn children, depend upon you.

C. EVERETT KOOP, M.D., SC.D.

Surgeon General

United States Public Health Service

\section{AIDS: A challenge for osteopathic physicians}

Information is abundant concerning the current and projected magnitude of this universal health threat called the acquired immune deficiency syndrome (AIDS). As each month passes, new concepts and facts become apparent requiring our constant attention, for it is a disease that will affect each of our lives and, for many, will alter the way we practice osteopathic medicine. The multiple psychosocial, legal, and ethical issues will demand our

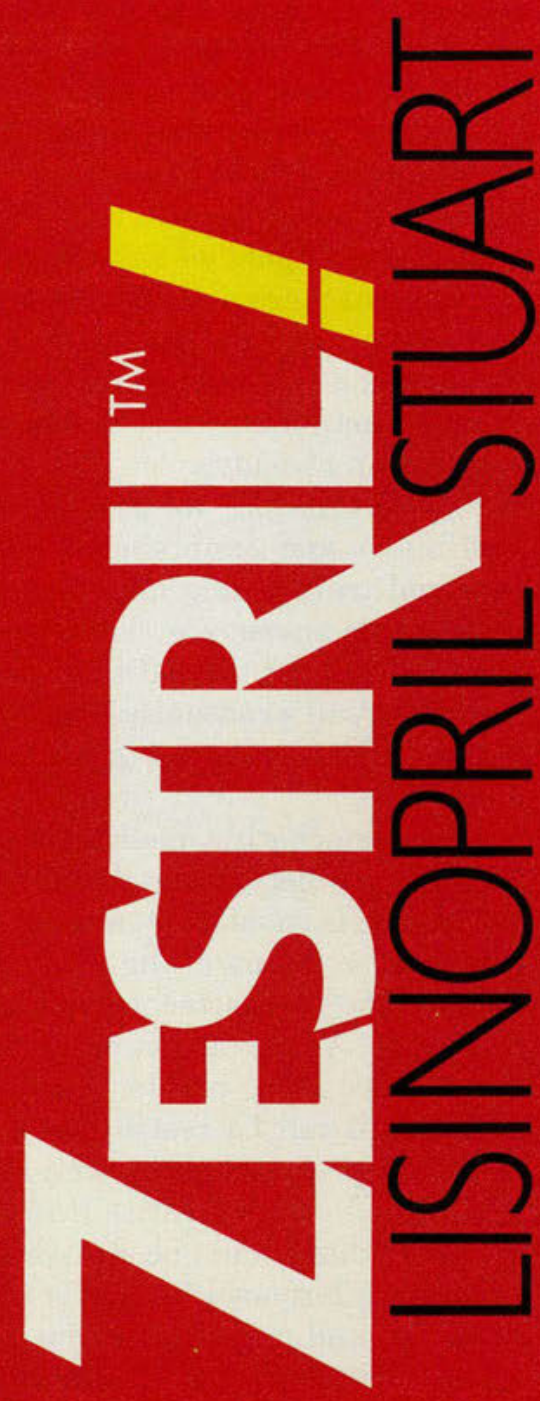

